\title{
Osteopontin stimulates melanoma growth and lung metastasis through NIK/MEKK1-dependent MMP-9 activation pathways
}

\author{
HEMA RANGASWAMI and GOPAL C. KUNDU
}

\author{
National Center for Cell Science (NCCS), Pune 411 007, India
}

Received April 4, 2007; Accepted May 10, 2007

\begin{abstract}
We examined the role of osteopontin (OPN) in NIKand MEKK1-dependent MMP-9 activation, melanoma growth and lung metastasis and its clinical significance in malignant melanoma. Here we report that OPN induces $\alpha \mathrm{v} ß 3$ integrinmediated MEKK1-dependent JNK1 phosphorylation. OPN stimulates NIK- or JNK1-dependent c-Jun expression. In contrast, OPN induces MEKK1-dependent JNK1 activation that leads to downregulation of ERK1/2 activation. OPN triggers NIK- and MEKK1-dependent AP-1 activation whereas NIK-dependent AP-1 activation is independent of JNK1 that leads to pro-MMP-9 activation. In vivo studies indicate that the levels of pNIK and MMP-9 are significantly higher in the OPN-induced primary tumor and metastasized lung compared to control. Clinical data revealed that the enhanced level of OPN and pNIK expression in the skin biopsies correlates with Clark's level and Breslow thickness. Altogether, OPN regulates negative cross-talk between NIK/ERK and MEKK1/ JNK1 pathways that controls melanoma progression.
\end{abstract}

\section{Introduction}

Osteopontin (OPN), is an RGD-containing non-collagenous, sialic acid-rich phosphoglycoprotein. It is a member of the extracellular matrix $(\mathrm{ECM})$ protein family $(1,2)$. It has an $\mathrm{N}$ terminal signal sequence and a highly acidic region consisting of nine consecutive aspartic acid residues (3). It binds with several integrins and CD44 variants in an RGD sequencedependent and -independent manner (4). This protein is involved in normal tissue remodeling processes such as bone resorption, angiogenesis, wound healing, and tissue injury as well as certain diseases such as tumorigenesis, restenosis, atherosclerosis and autoimmune diseases (5). OPN expression is upregulated in several cancers and is reported to associate with tumor progression and metastasis $(6,7)$. It has been reported that OPN induces MT1-MMP-mediated pro-matrix metalloproteinase-2 (pro-MMP-2) activation and urokinase

Correspondence to: Dr G.C. Kundu, National Center for Cell Science (NCCS), NCCS Complex, Pune 411 007, India

E-mail: gopalkundu@hotmail.com

Key words: osteopontin, NIK, MMP-9, melanoma growth, lung metastasis type plasminogen activator (UPA)-mediated pro-matrix metalloproteinase-9 (pro-MMP-9) activation, cell motility, ECM invasion and tumor growth (8-10).

MEKK1, a member of the MAP3K family is a mammalian serine/threonine protein kinase. It was initially identified on the basis of its homology with STE11 and activates the pheromone responsive MAPK cascade in yeast (11). Previous data indicated that overexpression of constitutively active forms of MEKK1 leads to JNK activation via phosphorylation of its upstream kinase, MKK4 (12). Nuclear factor inducing kinase (NIK) is another member of the MAP3K family that has been implicated in NFKB activation. Few reports indicate that NIK may also be involved in the regulation of transcription factor, AP-1 (13). Various MAPK cascades (e.g. ERK1/2, JNK, p38) are often portrayed as linear cascades and indications of crosstalk between the various cascades are limited (14). However, the cross-talk between NIK/ERK and MEKK1/JNK pathways in response to OPN that ultimately regulates melanoma growth and lung metastasis has not been well documented.

MMPs are extracellular matrix (ECM) degrading enzymes that play a critical role in embryogenesis, tissue remodeling, inflammation and angiogenesis (15). MMP-9, also referred to as type IV collagenase or gelatinase B, efficiently degrades native type IV and V collagens, fibronectin, ectactin and elastin and hence plays a major role in invasion, tumor growth and metastasis (16). MMP-9 has also been implicated in angiogenesis, rheumatoid arthritis, retinopathy and vascular stenosis and is considered to be a prioritized therapeutic target (17).

In this study, we have demonstrated that OPN induces $\alpha v ß 3$ integrin mediated NIK- and/or MEKK1-dependent cJun expression leading to AP-1 transactivation and MMP-9 activation in B16F10 cells. This OPN-induced MEKK1 and NIK-mediated AP-1 transactivation occurs through both JNKdependent and -independent pathways. OPN also induces a negative cross-talk between NIK/ERK and MEKK1/JNK1 pathways leading to MMP-9 activation that ultimately controls melanoma growth and lung metastasis. The levels of pNIK and MMP-9 are significantly higher in the OPN-induced metastasized lung compared to control. Clinical data revealed the enhanced level of OPN and pNIK expression in the skin biopsies that correlates with Clark's level and Breslow thickness that in turn reflects the melanoma grades.

\section{Materials and methods}

Cell culture. The B16F10 cells were cultured in Dulbecco's modified Eagle's medium (DMEM) as described previously (8). 
Table I. Analysis of OPN and pNIK expression and its correlation with metastatic potential in various grades of malignant melanoma specimens.

\begin{tabular}{lccccc}
\hline Case & Location & Type of MM & Breslow thickness (mm) & Clark's level & Metastasis \\
\hline 1 & Left foot & NM & 4 & IV & + \\
2 & Left second finger & NM & 6 & V & + \\
3 & Left foot & NM & 3 & III & + \\
4 & Lower lip & SSM & 2 & IV & - \\
\hline
\end{tabular}

MM, malignant melanoma; NM, nodular melanoma; and SSM, superficial spreading melanoma. $\mathrm{n}=3$ in each case.

In vivo tumorigenicity experiments. The tumorigenicity experiments were performed as described previously $(8,10)$. The cells were either treated with purified human OPN $(10 \mu \mathrm{M})$ or transfected with wild-type and kinase-negative NIK or wild-type and kinase-negative MEKK1 in the presence of Lipofectamine Plus and then treated with OPN and injected subcutaneously into the flanks of male athymic NMRI (nu/nu) mice. Six mice were used in each set of experiments. OPN $(10 \mu \mathrm{M})$ was again injected into the tumor sites twice a week for up to 4 weeks. After 4 weeks, the mice were sacrificed and the tumor weights were measured.

In vivo lung metastasis study. Cells were treated with OPN or stably transfected with wild-type and kinase-negative NIK and then treated with OPN, and then injected into the lateral tail veins of nude mice. The mice were sacrificed after eight weeks and the lungs were removed, washed with water and fixed. Sections of the lungs were stained with H\&E to confirm that the nodules were melanoma specific. The levels of expression of pNIK and MMP-9 were detected by immunostaining with their specific antibodies followed by FITC and analyzed by confocal microscopy (Zeiss).

Clinical specimens for immunohistochemical studies. Human primary melanoma tissue samples were collected with informed consent, from either diagnostic biopsies or upon surgery from a local hospital. Based on the clinical investigations, they were classified as malignant melanomas (MMs) with metastasis and MMs without metastasis. Clinical and histopathological data are summarized in Table I. Immunohistochemical studies were performed on the primary cutaneous lesions.

Plasmids and DNA transfection. The luciferase reporter construct (pAP-1-Luc) containing seven tandem repeats of the AP-1 binding site was from Stratagene. Cells were transiently transfected with cDNA using Lipofectamine Plus according to the manufacturer's instructions as described previously (9).

Immunoprecipitation and Western blot analysis. The levels of pMEKK1, MEKK1, pJNK1, JNK1, ERK, c-Jun and actin from treated or transfected $\mathrm{B} 16 \mathrm{~F} 10$ cell lysates or nuclear extracts were analyzed either by immunoprecipitation followed by Western blotting or direct Western blotting using their specific antibodies $(8,9)$.
In vitro kinase assay. The JNK1 or ERK kinase assays using c-Jun or myelin basic protein (MBP) as substrate were performed as described $(9,10)$.

AP-1 luciferase reporter gene assay. The AP-1 luciferase assays were performed by transfecting the cells with luciferase reporter construct (pAP-1-Luc) or cotransfecting with wildtype and kinase-negative NIK or wild-type and kinase-negative MEKK1 followed by treatment with OPN. The transfection efficiency was normalized by cotransfecting the cells with pRL vector. The luciferase activity was measured by a luminometer and changes in luciferase activity with respect to control were calculated.

Zymography. The gelatinolytic activity of MMP-9 in treated or transfected cells was measured as described (10).

\section{Results}

OPN induced $\alpha v \beta 3$ integrin-dependent MEKK1 and JNK1 phosphorylation. To study the effect of OPN on MEKK1 phosphorylation, B16F10 cells were treated with $5 \mu \mathrm{M}$ OPN at $37^{\circ} \mathrm{C}$. Cell lysates were immunoprecipitated with anti-MEKK1 antibody and immunoblotted with anti-phosphoserine antibody. The data revealed that OPN induces MEKK1 phosphorylation (Fig. 1A). In separate experiments, cells were either pretreated with anti- $\alpha \mathrm{v} B 3$ integrin antibody or transfected with mut NIK or wt and mut MEKK1 and then treated with OPN and used for JNK1 phosphorylation. avß3 integrin antibody or mut MEKK1 but not mut NIK suppressed the OPN-induced JNK1 phosphorylation in these cells (Fig. 1B). All the bands were quantified by densitometric analysis and the fold-changes were calculated. These results demonstrated that OPN induces MEKK1 and JNK1 phosphorylation through an $\alpha \mathrm{V} ß 3$ integrinmediated but NIK-independent pathway.

OPN-induced JNK kinase activity was regulated by MEKK1 but not by NIK. To examine the role of NIK and MEKK1 in regulating OPN-induced JNK1 activity, cells were transfected with wild-type and kinase-negative NIK or wild-type and kinase-negative MEKK1 and then treated with OPN. JNK1 kinase assay was performed. The data revealed that wild-type MEKK1 enhanced whereas mut MEKK1 suppressed the OPNinduced JNK activity (Fig. 1C). The data also indicated that OPN-induced JNK1 activity was unaffected upon transfection 


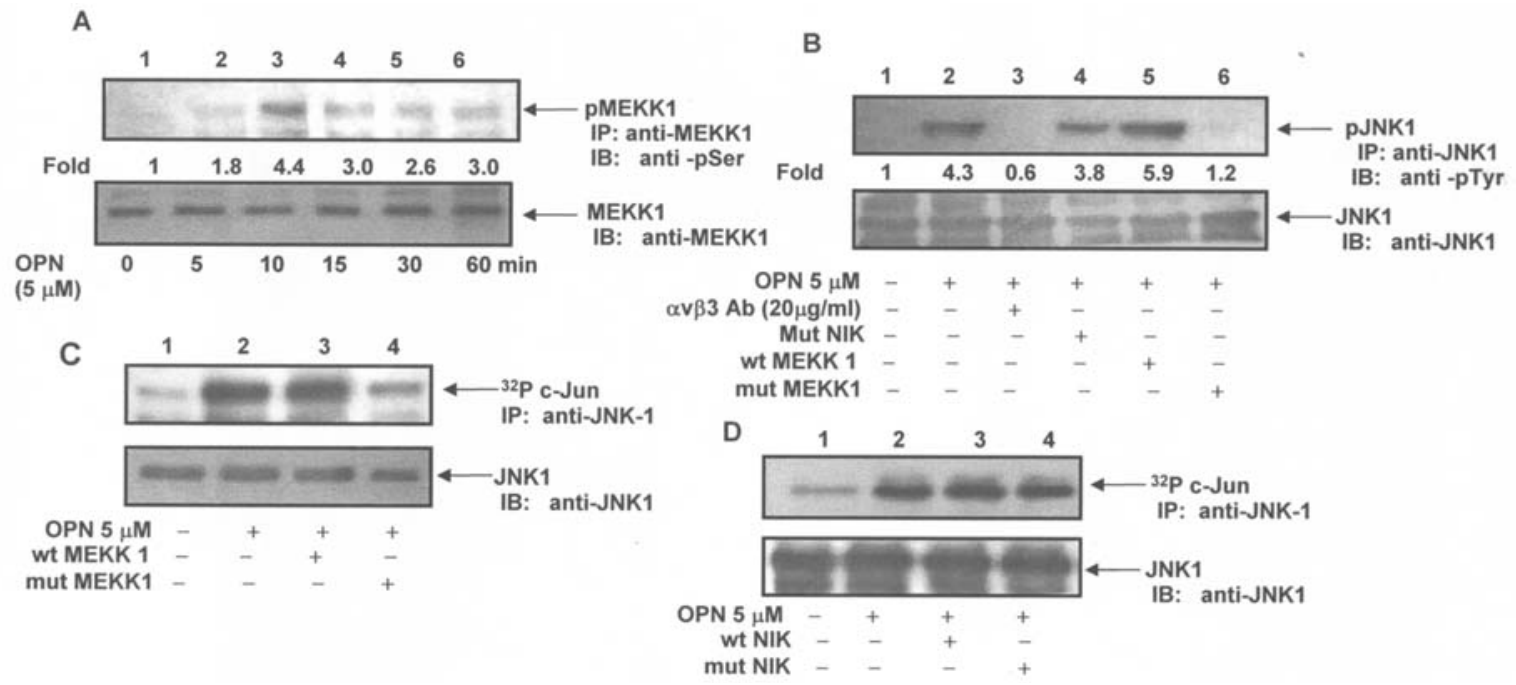

Figure 1. (A) OPN stimulated MEKK1 phosphorylation. B16F10 cells were treated with $5 \mu$ M OPN for 0-60 min. Cell lysates were immunoprecipitated with anti-MEKK1 antibody and immunoblotted with anti-phosphoserine antibody (upper panel). The same blot was reprobed with anti-MEKK1 antibody as loading control (lower panel). (B) OPN-induced avB3 integrin-mediated JNK1 phosphorylation was enhanced by MEKK1 but not by NIK. Cells were pretreated with anti- $\alpha \mathrm{vB3}$ integrin antibody or transfected with wt and mut MEKK1 or mut NIK and then stimulated with OPN. Cell lysates were immunoprecipitated with anti-JNK1 antibody and immunoblotted with anti-phosphotyrosine antibody (upper panel). The same blots were reprobed with anti-JNK1 antibody (lower panel). (C and D) OPN induced MEKK1-dependent (C) but NIK-independent (D) JNK1 activity. Cells were treated with $5 \mu$ M OPN or transfected with wt and mut MEKK1 or wt and mut NIK and then stimulated with OPN. Cell lysates were immunoprecipitated with anti-JNK1 antibody and JNK1 kinase assay was performed using recombinant c-Jun as substrate (upper panels). The remaining half of the immunoprecipitated samples was immunoblotted with anti-JNK1 antibody (lower panels) as controls. All these bands were analyzed densitometrically and the fold-changes were calculated. The data shown here represent three experiments exhibiting similar effects.

of cells with both wild-type and mut NIK (Fig. 1D). The same blots were reprobed with anti-JNK1 antibody as loading control. These data suggested that MEKK1 but not NIK regulates OPN-induced JNK1 activity.

Overexpression of active MEKK-1 attenuated OPN-induced ERK1/2 activation. MEKK-1 functions as a MAPKKK in the JNK pathway; however several reports have suggested that MEKK-1 may also affect the ERK pathway (14). Previous results indicated that $\mathrm{JNK}^{-/-}$mice showed enhanced ERK phosphorylation leading to tumor growth (18), therefore we speculated that activation of JNK1 by OPN may play a role in suppression of ERK1/2 activation. Accordingly, cells were transfected with wild-type and mut MEKK1 or wild-type JNK1 and then treated with OPN. In a separate experiment, cells were pretreated with SP600125 and then treated with OPN. ERK kinase activity was performed using MBP as a substrate. The data indicated that overexpression of wild-type MEKK1 and wild-type JNK1 completely attenuated OPNinduced ERK activation. Treatment with SP600125 partially suppressed the OPN-induced ERK activity (Fig. 2A). Half of the immunoprecipitated samples were immunoblotted with anti-ERK1/2 antibody. These results suggested that MEKK1 negatively regulates OPN-induced ERK activation.

OPN induced MEKKI- and NIK-mediated c-Jun expression. To determine the effect of OPN on c-Jun expression and to examine the role of MEKK1 and NIK in this process, cells were either treated with OPN or transfected with wt and mutant forms of NIK and MEKK1 and then treated with OPN. The level of c-Jun expression was detected by Western blotting using anti-c-Jun antibody. The results indicated that
OPN induced c-Jun expression (Fig. 2B). Wild-type NIK and MEKK1 enhanced whereas mut NIK and MEKK1 suppressed OPN-induced c-Jun expression. Actin was used as loading control. These data indicated that OPN induced c-Jun expression through both NIK- and MEKK1-dependent pathways.

OPN induced NIK- and MEKK1-dependent AP-1 transactivation. To further investigate the role of NIK and MEKK1 in $\mathrm{OPN}$-induced AP-1 transactivation, luciferase reporter gene assay was performed. Cells were either transfected with AP-1 luciferase reporter construct (pAP-1-Luc) or cotransfected with wild-type and mut NIK or wild-type and mut MEKK1 along with pAP-1-Luc and then treated with OPN. Cotransfecting the cells with pRL vector normalized the transfection efficiency. Changes in luciferase activity with respect to control were determined. The fold-changes are represented as a bar graph. The results are expressed as the means + S.E. of three determination. The values were also analyzed by Student's t-test $(\mathrm{p}<0.002)$. The data showed that wild-type NIK and MEKK1 enhanced but mut NIK and MEKK1 suppressed OPN-induced AP-1 activity in these cells (Fig. 2C). These data indicated that OPN induced AP-1 transactivation through NIK and MEKKmediated pathways.

OPN stimulated MEKK1-mediated MMP-9 activation. The effect of MEKK1 on OPN-induced MMP-9 activation was determined by zymography. Cells were transfected with wild-type and mut MEKK1 and then treated with OPN. The conditioned media were collected and used for gelatinolytic activity by zymography. An increased level of MMP-9 activation $(86 \mathrm{kDa})$ was observed when cells were treated with OPN 


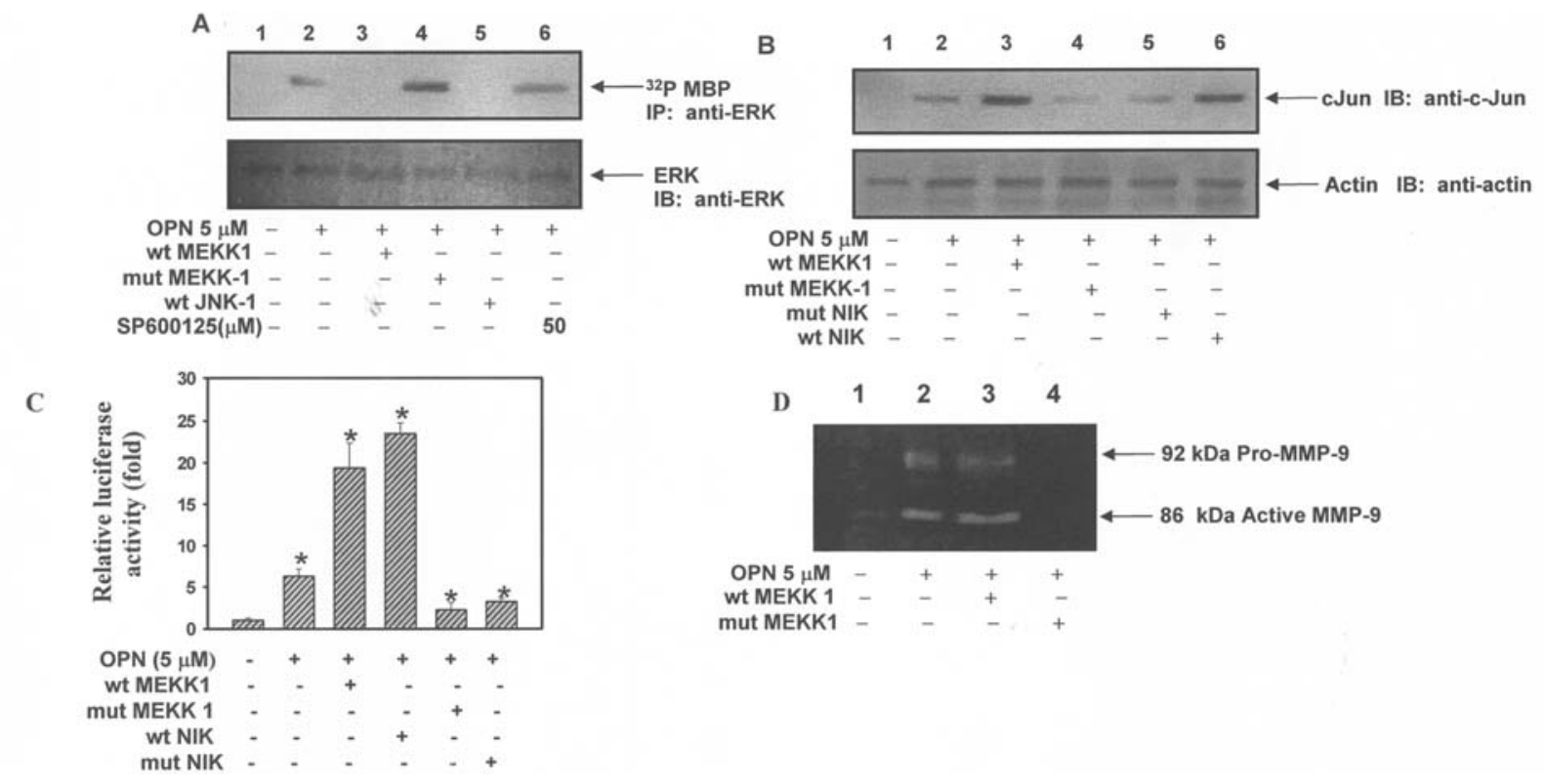

Figure 2. (A) Overexpression of MEKK1 suppressed OPN-induced ERK activation. Cells were transfected with wt and mut MEKK1 or wt JNK1 or pretreated with SP600125 and then treated with OPN. Cell lysates were immunoprecipitated with anti-ERK1/2 antibody and half of the immunoprecipitated samples were used for ERK kinase assay using MBP as a substrate (upper panel). Half of the immunoprecipitated samples were immunoblotted with anti-ERK1/2 antibody (lower panel). (B) OPN enhanced NIK- and MEKK1-dependent c-Jun expression. Cells were treated with $5 \mu \mathrm{M}$ OPN or transfected with wt and mut NIK or wt and mut MEKK1 and then treated with OPN. The level of c-Jun in the nuclear extracts was analyzed by Western blotting using anti-c-Jun antibody (upper panel). Actin was used as loading control (lower panel). The data shown here represent three experiments exhibiting similar effects. (C) OPN enhanced NIK- and MEKK1-dependent AP-1 transactivation. Cells were either transfected with luciferase reporter construct (pAP-1-Luc) or cotransfected with wt and mut NIK or wt and mut MEKK1 along with pAP-1-Luc and then treated with OPN. Cell lysates were used to measure the luciferase activity. The values were normalized to Renilla luciferase activity. The fold changes were calculated and mean \pm S.E. of triplicate determinations are plotted. The values were also analyzed by Student's t-test ( $\left.{ }^{*} \mathrm{p}<0.002\right)$. (D) OPN induced MEKK1-dependent pro-MMP-9 activation. Cells were transfected with wt and mut MEKK1 and then treated with OPN. The conditioned media were collected and activity of MMP-9 was examined by gelatin zymography. The data shown here represent three experiments exhibiting similar effects.

(Fig. 2D). Almost no MMP-9 specific band was detected in the untreated cells. Wild-type MEKK1 enhanced whereas mut MEKK1 suppressed OPN-induced MMP-9 activation.

OPN induced NIK/MEKK1-dependent melanoma growth in nude mice. The in vitro data prompted us to examine whether NIK and MEKK1 play any role in OPN-induced tumor growth in nude mice. Accordingly, cells were either treated with OPN or transfected with wild-type and mut NIK or wild-type and mut MEKK1 followed by treatment with OPN and then injected subcutaneously into the flanks of nude mice. The mice were sacrificed after four weeks and the tumor weights were measured. The fold-change was determined and is represented in the form of a bar graph (Fig. 3A). There was a 6- and 5.4-fold increase in tumor weight when wt NIK- or wt MEKK1-transfected cells were injected respectively. The tumor size was significantly less in mice injected with mut NIK- or mut MEKK1-transfected cells. The changes in tumor weights were also analyzed statistically by Student's t-test $(\mathrm{p}<0.002)$.

Role of NIK on melanoma metastasis to lungs. To examine the role of NIK in regulating OPN-induced MMP-9 expression in lung metastasis of melanoma, cells were stably transfected with wild-type and mut NIK and then treated with OPN and injected through the lateral tail vein into nude mice. The mice were sacrificed after 8 weeks and the lungs were removed and fixed in Bouin's solution for $24 \mathrm{~h}$ to facilitate counting of tumor nodules using a dissecting microscope. Fig. 3B shows that wild-type NIK increased the formation of metastatic nodules (lung colonies) compared to mutant NIK or OPN injected lungs. The levels of MMP-9 (Fig. 3B) and pNIK (Fig. 3C) were analyzed in lung sections by immunohistochemistry. The data indicated that the level of pNIK was significantly higher in OPN-treated metastasized lung compared to control and MMP-9 expression was significantly higher in wild-type NIK-transfected samples treated with OPN compared to OPN alone or mutant NIK-transfected cells treated with OPN. These data clearly demonstrated for the first time that pNIK and MMP-9 play a crucial role in OPN-regulated melanoma metastasis to lungs.

Expression of OPN and pNIK in human MM specimens. In order to correlate the in vitro data and in vivo mouse model results to human clinical specimens, primary melanoma tissues were collected with informed consent, from either diagnostic biopsies or upon surgery. The levels of expression of OPN and pNIK were determined by immunohistochemical analysis. The results revealed that OPN and pNIK were present in human malignant melanoma specimens of all 12 patients with four different cases (3 MM with metastasis and $1 \mathrm{MM}$ without metastasis). Moreover, there was significant positive correlation between the expression of OPN and pNIK and melanoma progression in human specimens of all four cases (Fig. 4). Clinical and histopathological data are summarized in Table I. 


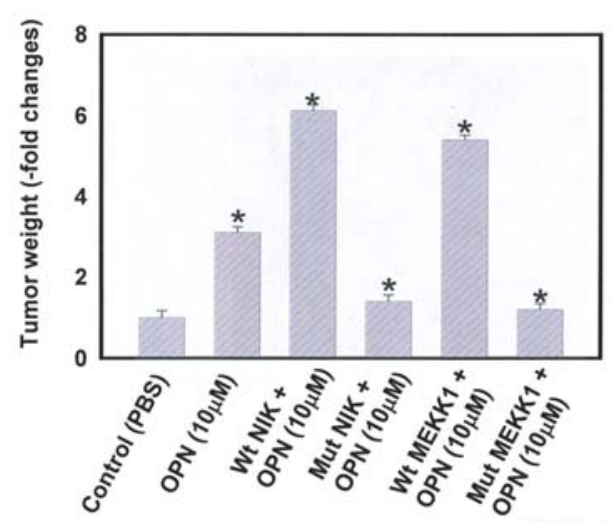

Control

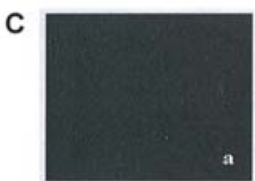

B
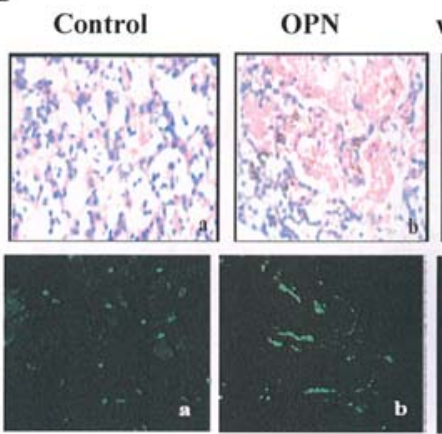

wt NIK + OPN

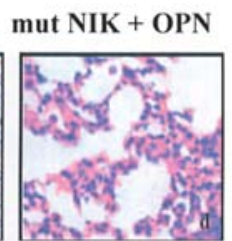

H\&E
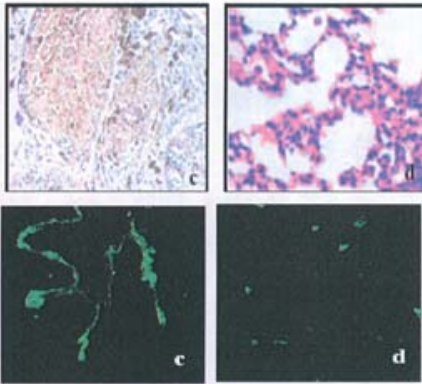

IF: MMP-9

OPN

IF: pNIK

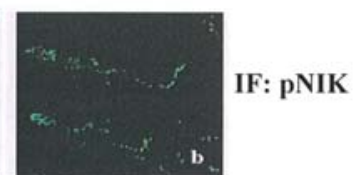

Figure 3. (A) NIK and MEKK1 play a crucial role in OPN-induced tumor growth in nude mice. Cells treated with OPN or transfected with wt and mut NIK or wt and mut MEKK1 were injected subcutaneously into nude mice. After 4 weeks, mice were sacrificed and tumor weights were measured. Bar graph depicting the changes in tumor weight. Mice injected with cells in PBS were used as controls. The changes in tumor weights were also analyzed statistically by Student's t-test $(\mathrm{p}<0.002) ; \mathrm{n}=6$ (each group). (B) Pivotal role of NIK in OPN-induced melanoma metastasis to lungs. The role of NIK in OPN-induced melanoma metastasis to lungs was studied. Note that large metastatic nodules were formed in both OPN-injected and wt NIK expressing cells treated with OPN when compared to the mice injected with mut NIK-transfected cells (x20). Photograph of H\&E sections. Note that more dark spots (melanin pigments) in wt NIK-transfected metastasized lungs of H\&E-stained sections were observed. Immunofluorescence staining of MMP-9 in the control (a), OPN-treated (b), wt NIK (c) and mut NIK (d) stably expressed OPN-treated lung sections was performed. (C) Immunofluorescence staining of pNIK in metastasized lung sections indicated that the level of pNIK was significantly higher in metastasized lung induced by OPN compared to the control (x60). $\mathrm{n}=6$ (each group).

Case 1

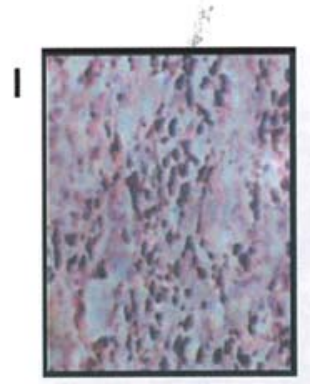

II

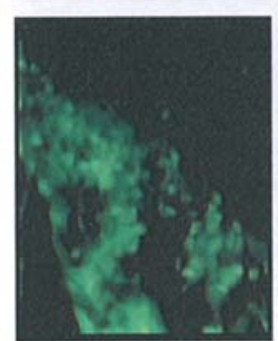

III

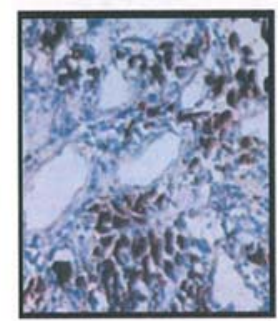

Case 2
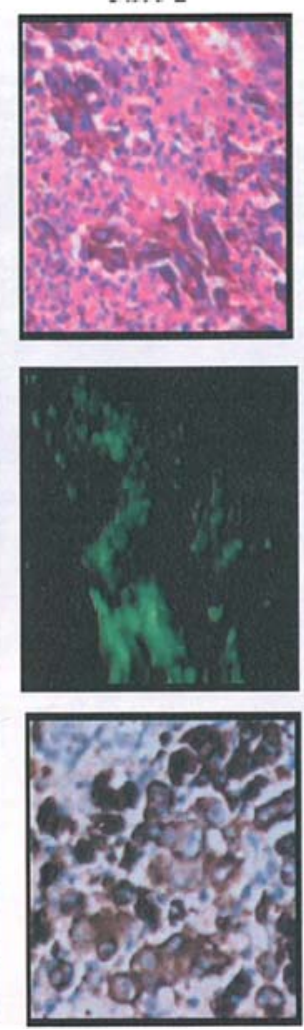

Case 3
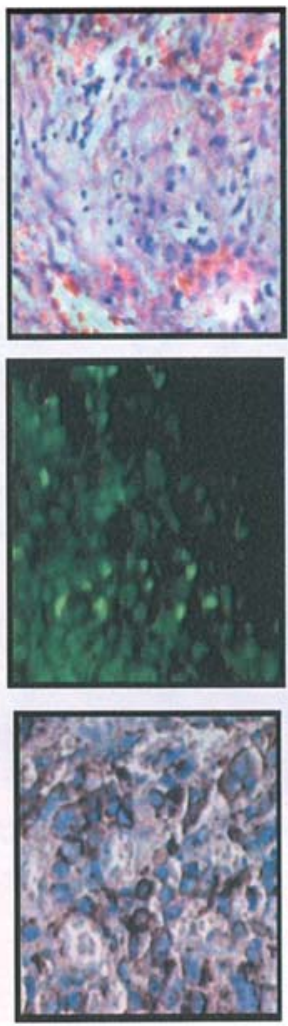

Case 4

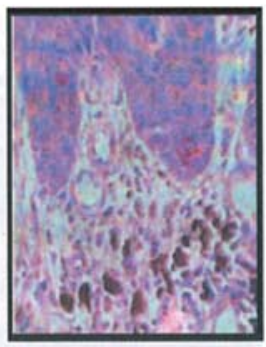

$H \& E$

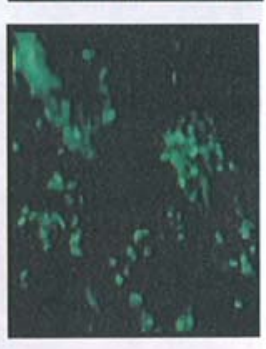

OPN

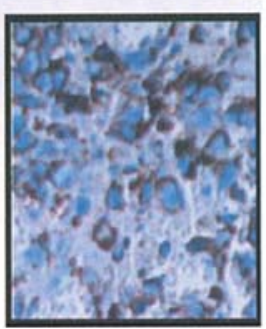

pNIK

Figure 4. Correlation of OPN and pNIK expression with melanoma progression in human clinical specimens. Immunohistochemical studies were performed on the primary cutaneous lesions and Clark's level and Breslow thickness were determined. The levels of OPN and pNIK expression were determined by immunofluorescence staining using the respective primary antibodies followed by either FITC- or HRP-conjugated IgG and analyzed using confocal microscopy. Note that there was a significant positive correlation between OPN and pNIK expression and metastatic potential in different grades of melanoma specimens. These clinical and histopathological data are summarized in Table I. 


\section{Discussion}

Recent studies have demonstrated that downregulation of NIK activation does not affect TNF $\alpha$-induced JNK activation (19). It has been also reported that ERK and JNK pathways play a crucial role in regulating MMP-9 activation and cell motility in growth factor stimulated human epidermal keratinocytes (20). These results prompted us to investigate whether binding of OPN to $\alpha \mathrm{v} ß 3$ integrin receptor regulates JNK1 activation and whether NIK is involved in this activation process. We have shown that OPN induces avß3 integrinmediated MEKK1 and JNK1 phosphorylation whereas JNK1 phosphorylation is MEKK1-dependent but NIK-independent. Shen et al have recently reported that sustained activation of JNK blocks ERK activation in response to EGF and PMA (21). Our data demonstrated a negative cross-talk between OPN-induced NIK/ERK and MEKK1/JNK1 activation and further suggested that sustained activation of JNK resulted in the attenuation of ERK activation. Previous studies have indicated that MEKK1 has the ability to activate ERK but the effect is less potent (22). This may be implicated to the short and long phase of MEKK1 activation, which results in different cellular responses, a short-phase activation which leads to ERK activation and long-phase activation resulting in inhibition of ERK activation. Also the inhibition of OPN-induced NIKmediated ERK activation caused by overexpression of wildtype MEKK1 involves the ability of MEKK1 to activate the JNK pathway. These implications delineate a mechanism in which treatment with the same agonist may result in a different cellular outcome depending on the duration of treatment. This data is consistent with the recent report that JNK1 deficiency stimulates TPA-induced ERK phosphorylation leading to enhanced skin tumorigenesis (18). Natoli et al have reported that overexpression of NIK, which does not activate JNK, strongly activates transcription directed by a canonical AP-1 site (19). Because we have shown that OPN induces MEKK1dependent but NIK-independent JNK phosphorylation and the AP-1 response element is present in the promoter region of the MMP-9 gene, we sought to determine the level of c-Jun expression upon OPN stimulation. Our data indicated that OPN induces both NIK- and MEKK1-mediated c-Jun expression leading to AP-1 transactivation. Overexpression of NIK did not affect JNK activation but significantly upregulated AP-1 transcriptional activity indicating that OPN induces NIKdependent AP-1 activation, which is independent of JNK. In summary, we have reported that OPN induces pro-MMP-9 activation through NIK/ERK and MEKK1/JNK-mediated AP-1 dependent pathways. The in vitro data is also supported by in vivo findings, which showed that OPN enhances both NIK- and MEKK1-mediated tumor growth and lung metastasis in nude mice. The levels of pNIK and MMP-9 are significantly higher in the primary tumor and metastasized lung compared to control. Clinical data revealed the enhanced level of OPN and pNIK expression in the skin biopsies, which correlated with Clark's level and Breslow thickness that in turn, reflected the melanoma grade. These results highlight the potential role of OPN and the molecular mechanism underlying the genesis of metastasis signature in melanoma and hence may be useful in developing novel molecular diagnostics and targeted therapy for the treatment of malignant melanoma.

\section{Acknowledgments}

We thank Professor David Wallach for providing wild-type NIK (wt pcDNA NIK) and kinase-negative NIK (mut pcDNA NIK; NIK-KK429/430AA) in pcDNA3 and the wild-type and dominant negative constructs of MEKK1 (pcDNA3-MEKK1 and pcDNA3-FlagMEKK1 K432M) were kind gifts from Professor Tom Maniatis (Harvard University, Cambridge). The wild-type JNK1 in pcDNA3 was a kind gift from Dr Roger Davis (University of Massachusetts Medical School, Worcester).

\section{References}

1. Butler WT: The nature and significance of Osteopontin. Connect Tissue Res 23: 123-136, 1989.

2. Denhardt D and Guo X: Osteopontin: a protein with diverse functions. FASEB J 7: 1475-1482, 1993.

3. Prince CW: Secondary structure predictions for rat Osteopontin. Connect Tissue Res 21: 15-20, 1989.

4. Weber GF, Akshar S, Glimcher MJ and Cantor H: Receptorligand interaction between CD44 and osteopontin (Eta-1). Science 271: 509-519, 1996.

5. Liaw L, Birk DE, Ballas CB, Whitsitt JS, Davidson JM and Hogan BL: Altered wound healing in mice lacking a functional osteopontin gene. J Clin Investig 101 (suppl 1): 1468-1478, 1998.

6. Brown LF, Papadopoulos-Sergiou A, Berse B, Manseau EJ, Tognazzi K, Perruzzi CA, Dvorak HF and Senger DR: Osteopontin expression and distribution in human carcinomas. Am J Pathol 145: 610-623, 1994.

7. Craig AM, Bowden GT, Chambers AF, Spearman MA, Greenberg AH, Wright JA and Denhardt DT: Secreted phosphoprotein mRNA is induced during multi-stage carcinogenesis in mouse skin and correlates with the metastatic potential of murine fibroblasts. Int J Cancer 46: 133-137, 1990.

8. Philip S and Kundu GC: Osteopontin induces nuclear factor kappa B-mediated promatrix metalloproteinase- 2 activation through I kappa B alpha/IKK signaling pathways, and curcumin (diferulolylmethane) downregulates these pathways. J Biol Chem 278: 14487-14497, 2003.

9. Das R, Mahabeleshwar GH and Kundu GC: Osteopontin induces AP-1-mediated secretion of urokinase-type plasminogen activator through c-Src-dependent epidermal growth factor receptor transactivation in breast cancer cells. J Biol Chem 279: 11051-11064, 2004.

10. Rangaswami H, Bulbule A and Kundu GC: Nuclear factorinducing kinase plays a crucial role in osteopontin-induced $\mathrm{MAPK} / \mathrm{I} \kappa \mathrm{B} \alpha$ kinase dependent nuclear factor $\kappa \mathrm{B}$-mediated promatrix metalloproteinase-9 activation. J Biol Chem 279: 38921-38935, 2004.

11. Lange-Carter CA, Pleiman CM, Gardner AM, Blumer KJ and Johnson GL: A divergence in the MAP kinase regulatory network defined by MEK kinase and Raf. Science 260: 315-319, 1993.

12. Yan M, Dai T, Deak JC, Kyriakis JM, Zon LI, Woodgett JR and Templeton DJ: Activation of stress-activated protein kinase by MEKK1 phosphorylation of its activator SEK1. Nature 372: 798-800, 1994.

13. Karin M: The regulation of AP-1 activity by mitogen-activated protein kinases. Philos Trans R Soc Lond Biol Sci 351: 127-134, 1996.

14. Gallo KA and Johnson GL: Mixed-lineage kinase control of JNK and p38 MAPK pathways. Nat Rev Mol Cell Biol 3: 663-672, 2002.

15. Shapiro SD: Matrix metalloproteinase degradation of extracellular matrix: biological consequences. Curr Opin Cell Biol 10: 602-608, 1998.

16. Scorilas A, Karameris A, Amogiannaki N, Ardavanis A, Bassilopoulos P, Trangas $\mathrm{T}$ and Talleri M: Overexpression of matrix-metalloproteinase- 9 in human breast cancer, a potential favourable indicator in node-negative patients. Br J Cancer 84: 1488-1496, 2001.

17. Folkman J: Angiogenic zip code. Nat Biotechnol 17: 749, 1999.

18. She Q, Chen N, Bode AM, Flavell RA and Dong Z: Deficiency of c-Jun-NH(2)-terminal kinase-1 in mice enhances skin tumor development by 12-O-tetradecanoylphorbol-13-acetate. Cancer Res 62: 1343-1348, 2002. 
19. Natoli G, Costanzo A, Moretti F, Fulco M, Balsano C and Levrero M: Tumor necrosis factor (TNF) receptor 1 signaling downstream of TNF receptor-associated factor 2. Nuclear factor kappaB (NFkappaB)-inducing kinase requirement for activation of activating protein 1 and NFkappaB but not of c-Jun Nterminal kinase/stress-activated protein kinase. J Biol Chem 272: 26079-26082, 1997.

20. Zeigler ME, Chi Y, Schmidt T and Varani J: Role of ERK and JNK pathways in regulating cell motility and matrix metalloproteinase 9 production in growth factor-stimulated human epidermal keratinocytes. J Cell Physiol 180: 271-284, 1999.
21. Shen YH, Godlewski J, Zhu J, Sathyanarayana P, Leaner V, Birrer MJ, Rana A and Tzivion G: Cross-talk between JNK/SAPK and ERK/MAPK pathways: sustained activation of JNK blocks ERK activation by mitogenic factors. J Biol Chem 278: 26715-26721, 2003

22. Minden A, Lin A, McMahon M, Lange-Carter C, Derijard B, Davis RJ, Johnson GL and Karin M: Differential activation of ERK and JNK mitogen-activated protein kinases by Raf- 1 and MEKK. Science 266: 1719-1723, 1994. 